\title{
Detection of coronary complications after the arterial switch operation for transposition of the great arteries: First experience with multislice computed tomography in children
}

Phalla Ou, MD, ${ }^{\mathrm{a}, \mathrm{b}}$ Elie Mousseaux, MD, PhD, ${ }^{\mathrm{c}}$ Arshid Azarine, MD, ${ }^{\mathrm{c}}$ Peggy Dupont, MD, ${ }^{\mathrm{b}}$ Gabriella Agnoletti, MD, Pascal Vouhé, MD, PhD, ${ }^{d}$ Daniel Sidi, MD, PhD, and Damien Bonnet, MD, PhD ${ }^{\mathrm{b}}$

Objective: The main cause of long-term morbidity and mortality after a successful arterial switch operation for transposition of the great arteries is complications at the ostial segments, proximal segments, or both of the retransferred coronary arteries. The purpose of this study was to investigate the clinical usefulness of multislice computed tomographic angiography in detecting ostial and proximal coronary lesions in children having undergone the arterial switch operation for transposition of the great arteries.

Methods: Forty-nine children (aged $8.5 \pm 3.9$ years) operated on for transposition of the great arteries with the arterial switch operation (follow-up, $8.3 \pm 3.6$ years) underwent systematic selective conventional and multislice computed tomographic angiography. The ability of multislice computed tomography in detecting stenosis and other modifications of the coronary arteries' course was analyzed by 2 independent investigators.

Results: Multislice computed tomography, as compared with selective conventional coronary angiography, permitted assessment of ostial and proximal coronary segments in every patient. It correctly detected the $4(8.1 \%)$ patients with significant coronary lesions ( 1 with ostial and 3 with proximal coronary stenosis) that had been identified by means of conventional angiography. Multislice computed tomography clearly showed an abnormal course of the coronary artery between the great arteries with compression of the ostial (1 patient) and proximal ( 3 patients) segments of the retransferred coronary arteries.

From the Service de Radiologie Pédiatrique ${ }^{\mathrm{a}}$ and Service de Cardiologie Pédiatrique, ${ }^{\text {b }}$ the Service de Radiologie Cardiovasculaire, Hôpital Européen Georges Pompidou, ${ }^{c}$ Hôpital Necker-Enfants Malades; and the Service de Chirurgie Cardiaque Pédiatrique, ${ }^{\mathrm{d}}$ Hôpital Necker-Enfants Malades, Paris, France.

Received for publication Sept 12, 2005; revisions received Oct 30, 2005; accepted for publication Nov 8, 2005.

Address for reprints: Phalla Ou, MD, Service de Radiologie Pédiatrique, Hôpital NeckerEnfants Malades, 149, rue de Sèvres, 75743 Paris Cedex 15, France (E-mail: phalla.ou@ nck.ap-hop-paris.fr).

J Thorac Cardiovasc Surg 2006;131:639-43

$0022-5223 / \$ 32.00$

Copyright $(9) 2006$ by The American Association for Thoracic Surgery

doi:10.1016/j.jtcvs.2005.11.014
Conclusion: These results indicate that multislice computed tomographic angiography is fully accurate in detecting ostial coronary artery stenoses, proximal coronary artery stenoses, or both in pediatric patients having undergone the arterial switch operation for transposition of the great arteries. Our results suggest that multislice computed tomography could be used as a screening technique for detecting coronary complications in the follow-up of the arterial switch operation before having recourse to conventional angiography.

$\mathrm{T}$ The main cause of long-term morbidity and mortality after the arterial switch operation (ASO) for transposition of the great arteries (TGA) is complications at the ostial segments, proximal segments, or both of the retransferred coronary arteries. ${ }^{1-7}$ Severe coronary lesions, including stenosis, compression, kinking, or occlusion, might be symptom free. Current noninvasive examinations, such as electrocardiography, exercise testing, echocardiography, and myocardial scintigraphy, are not sensitive enough to screen patients at high risk for myocardial infarction or sudden death. ${ }^{7}$ As a result, selective conventional angiography is system- 


\author{
Abbreviations and Acronyms \\ ASO = arterial switch operation \\ $\mathrm{CT}=$ computed tomography \\ MRI = magnetic resonance imaging \\ MSCT $=$ multislice computed tomography \\ $\mathrm{TGA}=$ transposition of the great arteries
}

atically proposed as the standard technique for detecting coronary lesions after the ASO, ${ }^{2,3,7}$ although it is invasive and potentially harmful.

In contrast, multislice computed tomography (MSCT) is an adequate and noninvasive diagnostic tool that has proved promising in detecting proximal coronary atheromatous stenosis in adults. ${ }^{8,9}$ However, the pertinence of using MSCT coronary angiography after the ASO in pediatric patients has yet to be established. To our knowledge, no previous studies have examined the potential usefulness of MSCT angiography in detecting ostial coronary complications, proximal coronary complications, or both in children who had undergone the ASO for TGA.

The aim of this study is to demonstrate the ability of MSCT angiography, as compared with selective conventional angiography, in detecting high-grade ostial coronary stenosis, proximal coronary stenosis, or both in children undergoing the ASO for TGA.

\section{Patients and Methods Patients}

A total of 49 pediatric patients undergoing the ASO for TGA were included in the study (age: mean of $8.5 \pm 3.9$ years, median of 9 years, and range of 5-10 years; weight: mean of $26 \pm 5 \mathrm{~kg}$, median of $24 \mathrm{~kg}$; age at operation: mean of $7 \pm 5$ days; follow-up after the operation: mean of $10.4 \pm 4.6$ years, median of 9 years, and range of 5-10 years). All patients were clinically well without any medication. Every patient referred to our department for systematic conventional coronary angiography was screened for possible participation. If there was no contraindication to $\beta$-blockers or increased serum creatinine level and informed consent was given by parents or tutors, the patient was included in the study. The mean interval between examinations was less than 1 month. The hospital ethics committee approved this study.

\section{Conventional X-ray Angiography Protocol}

Catheterization was done after achievement of sedation and local anesthesia with the transfemoral approach, as previously described. ${ }^{3}$ Selective injection into the coronary arteries was performed manually. Particular attention was paid to the opacification of each coronary artery orifice, and coronary artery angiograms were considered satisfactory only when good opacification of the coronary artery ostium was obtained. Biplane angiograms were performed in lateral, left, and right anterior oblique views $\left(20^{\circ}\right)$ to evaluate the coronary artery arising from the left and right sides of the neoaortic root.

\section{MSCT Scan Protocol}

MSCT angiography was performed with a 4-detector-row MSCT scanner (Somatom Volume Zoom; Siemens Medical System, Forchheim, Germany). All children received an oral sedative (hydroxyzine, $1 \mathrm{mg} / \mathrm{kg} 1$ hour before the examination, maximum of $100 \mathrm{mg}$ ) and a $\beta$-blocker (propranolol, $1 \mathrm{mg} \cdot \mathrm{kg}^{-1} \cdot \mathrm{d}^{-1}$ orally 48 hours before the examination). An additional $\beta$-blocker was administered in case of insufficient heart rate reduction ( $>80$ beats/min) in 10 (20.4\%) patients. A bolus of $2 \mathrm{~mL} / \mathrm{kg}$ intravenous nonionic contrast material (iobitridol [Xenetix]; $350 \mathrm{mg}$ iodine per $\mathrm{mL}$; Guerbet, Villepinte, France) was administrated through the antecubital vein with an automatic injector (Medrad En Vision CT, Pittsburgh, $\mathrm{Pa}$ ) at a flow rate of $3 \mathrm{~mL} / \mathrm{s}$. At the start delay of the scan fixed at 22 seconds, patients older than 6 years of age $(n=38,77.5 \%$ of patients) were automatically instructed to inspire and hold their breath during the computed tomographic (CT) data acquisition. Younger patients $(\mathrm{n}=11,22.5 \%$ of patients) were allowed to breath freely, although cries and agitation caused artifacts in 2 cases.

A contrast-enhanced retrospectively electrocardiogram-gated scan was obtained by using the following protocol: $4 \times 1-\mathrm{mm}$ slice collimation, table speed of $1.5 \mathrm{~mm}$ per rotation (rotation time, $500 \mathrm{~ms}$ ), and effective tube current of between 200 to $300 \mathrm{mAs}$ at $120 \mathrm{kV}$ depending on the patient weight, leading to an estimated radiation exposure of between 8 and $10 \mathrm{mSv}$. CT imaging started $2 \mathrm{~cm}$ above the pulmonary artery bifurcation and stopped at the diaphragm caudally of all cardiac structures. Retrospective electrocardiogram-gated images were reconstructed in the diastolic and systolic phases of the cardiac cycle (at least 3 data sets: $30 \%$, $60 \%$, and $80 \%$ of the RR interval). If indicated, additional window positions were explored at $40 \%, 50 \%$, and $70 \%$ of the RR interval. Effective slice thickness was $1.25 \mathrm{~mm}$, and the reconstruction increment was $0.6 \mathrm{~mm}$. When possible (heart rate of $<80$ beats/ min), axial slices were reconstructed from the acquired MSCT data with the use of an algorithm that uses only the data from a half-gantry rotation $(250 \mathrm{~ms})$. A bisegmental reconstruction algorithm was used when heart rates were greater than 80 beats/min by using data from a 125-ms temporal window in 2 consecutive cardiac cycles.

\section{Data Analysis}

MSCT data sets were analyzed by 2 independent investigators (1 radiologist and 1 cardiologist) with experience in cardiac CT, including experience with congenital heart diseases, who were blinded to the result of conventional coronary angiography and blinded to any other information obtained by the family members. The coronary arteries were examined by using the axial slices and, when necessary, postprocessing tools, such as multiplanar reconstruction and maximum-intensity thin-slab projection. Only the ostial and proximal coronary segments were analyzed: the ostia of the left and right coronary arteries; the left main coronary artery, including its bifurcation to the left anterior descending coronary artery and the circumflex artery; and the proximal segment of the right coronary artery. Thus, 4 segments for each patient were localized and classified by visual estimation as being assessable or not. Then the assessable segments were qualified as normal or significantly stenosed ( $>50 \%$ coronary artery diameter reduction). Cases of disagreement were settled by a joined consensus reading. 


\section{Statistical Analysis}

All data were analyzed with JMP software, version 5.0.1a (SAS Institute Inc, Cary, NC). All data are expressed when appropriate in terms of the mean value $\pm \mathrm{SD}$, the range, and the median. MSCT results for coronary artery assessment were then compared with results of conventional angiography, which is considered a standard measure. Concordance between observers was calculated and expressed by the $\kappa$ value.

\section{Results}

Selective coronary angiography was successfully performed in 47 patients. The 2 other patients had nonselective angiography. However, opacification of the coronary ostia and proximal segments was good enough for including these 2 patients in the study.

MSCT was performed without complications in all patients. The mean heart rate at the time of contrast medium injection was $73.8 \pm 13.9$ beats $/ \mathrm{min}$, with a range of 55 to 105 beats/min. Ten $(20.4 \%)$ patients had a heart rate of more than 80 beats/min. Artifacts caused by agitation were observed in 2 patients, particularly when the breath-hold maneuver was not possible. However, the results for these patients were still included in the analysis.

By use of selective conventional angiography, all coronary segments were available for analysis. Four $(8.1 \%)$ patients had significant coronary stenoses, including 1 ostial and 3 proximal lesions.

MSCT, as compared with selective conventional coronary angiography, permitted assessment of ostial and proximal coronary segments in every patient. It correctly detected the 4 patients with significant coronary lesions that had been identified by conventional angiography. Interestingly, MSCT showed an abnormal course of the coronary artery between the great arteries in these 4 patients. The first patient had a single coronary ostium. The coronary lesion was ostial and was due to compression of the coronary artery as it passes between the neoaorta and the left pulmonary branch (Figure 1). The 3 other patients had proximal stenosis also caused by compression of the coronary artery that passes between the neoaorta and the pulmonary trunk (Figure 2). Although these peculiar relationships of the coronary arteries to the great arteries were clearly seen on MSCT, they were misdiagnosed on conventional angiography. Concordance between both MSCT observers was perfect $(\kappa$ value $=1)$.

\section{Discussion}

As previously reported, ${ }^{1-7}$ we found a relatively high incidence $(8.1 \%)$ of proximal coronary artery lesions at late follow-up. We also found that all cases of significant stenosis were located in the ostial segments, proximal segments, or both of the coronary arteries. Thus screening coronary lesions is important because they are not rare and constitute the major cause of late death. ${ }^{3,7}$ In addition,

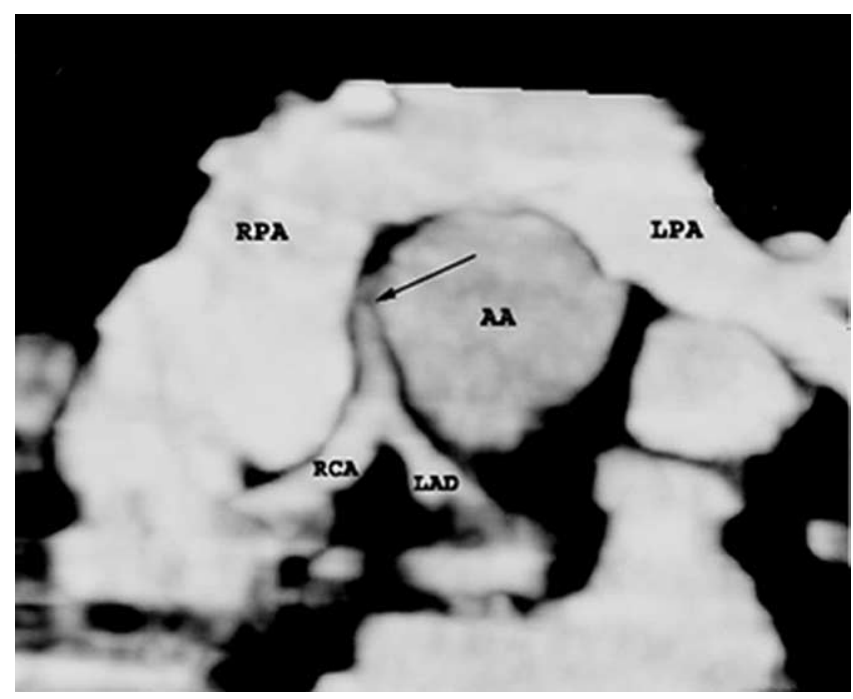

Figure 1. Ostial compression (arrow) of the unique coronary artery between the neoascending aorta and the right pulmonary artery. $R C A$, Right coronary artery; $L A D$, left anterior descending coronary artery; $R P A$, right pulmonary artery; $L P A$, left pulmonary artery; $A A$, neoascending aorta.

symptoms cannot be relied on because they are often absent in our population, as has been observed in other studies of late surgical follow-up. ${ }^{1,2,7}$

In this first study of MSCT coronary angiography in pediatric patients, we have shown that MSCT can be useful in follow-up of the ASO because it accurately detected ostial and proximal segments of the transferred coronary arteries. Indeed, the 4 patients presenting with coronary stenosis had their stenoses successfully detected. Therefore MSCT could be used as a safe and noninvasive screening technique to avoid repetitive coronary artery angiography.

In our study, although relatively outdated, 4-detector-row MSCT has been shown to be very accurate in detecting all ostial and proximal lesions, despite the smaller size of the coronary arteries and the higher heart rate observed in children compared with adults. The accuracy of MSCT was found to be better in our study compared with that previously reported in echocardiography, exercise testing, and myocardial scintigraphy, whether applied individually, in association, or both. ${ }^{7}$ Although the new generations of scanner with 16-, 32-, and 64-detector-row CT are emerging for use in clinical cardiovascular practice in adult patients, ${ }^{10,11}$ our study has interestingly demonstrated that the relatively outdated 4-detector-row scanner is sufficient in our specific pediatric patients who had undergone the ASO. Indeed, lesions of the retransferred coronary arteries are peculiar because they strictly differ from the atheromatous coronary disease in adults. Coronary lesions after the ASO are due to fibrocellular proliferation at the ostial scars; stretching, kinking 


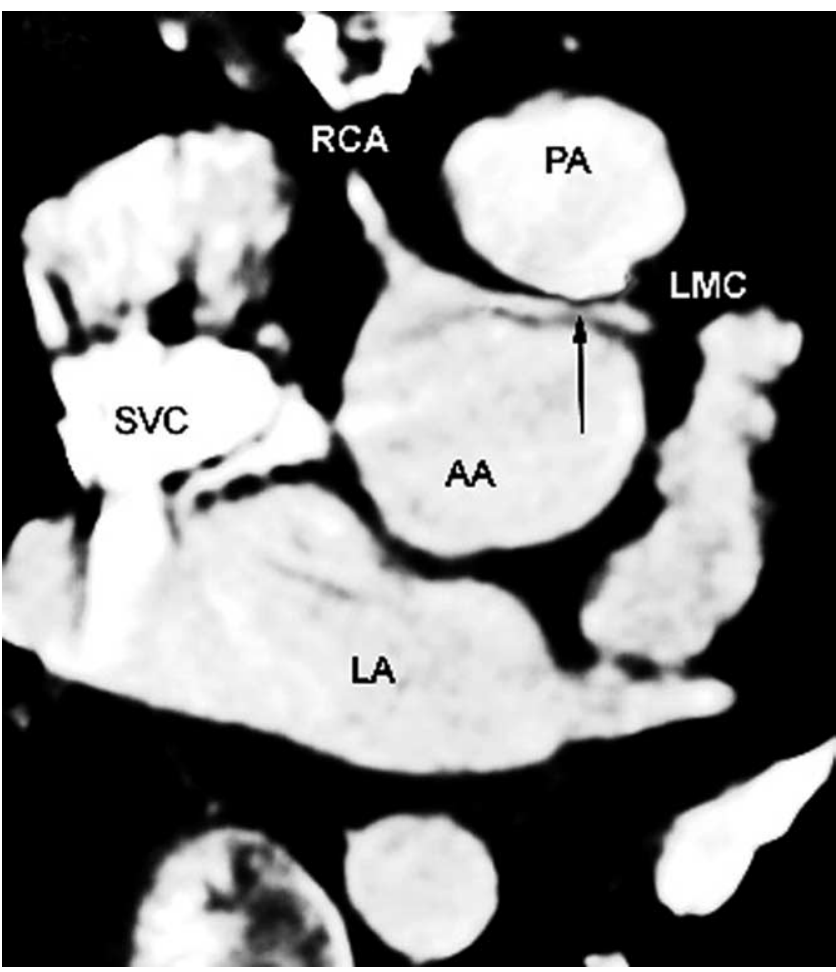

Figure 2. Compression of the left main coronary artery (arrow) between the neoascending aorta and the pulmonary trunk. $L M C$, Left main coronary artery; $R C A$, right coronary artery; $P A$, pulmonary artery; $A A$, neoascending aorta; $L A$, left atrium; $S V C$, superior vena cava.

or compression of proximal segments during growth; or both. $^{2}$ They exclusively concern the ostial segments, proximal segments, or both that are easily and accurately depicted on 4-detector-row scanner. We did not need to assess more distal coronary artery segments in our patients.

Compared with selective coronary artery angiography, MSCT has been shown to be superior to conventional angiography in the diagnosis of congenital anomalies of the coronary arteries, with accurate visualization of abnormal vessels in relation to the great mediastinal arteries. ${ }^{12}$ In the present study MSCT has the advantage of allowing a better understanding of mechanisms causing coronary artery stenosis because it permits good visualization of the environment near the vessel and the winding path of coronary arteries in and around the great vessels on the way to their respective cardiac grooves (Figures 1 and 2).

The major disadvantage of MSCT angiography is an x-ray radiation dose of 8 to $10 \mathrm{mSv}$. This is due to continuous acquisition of 4 axial slices during the entire cardiac cycle when only diastolic data are used. Recent studies have been performed with prospectively electrocardiogram-controlled roentgen tube modulation. ${ }^{13}$ Thus a $50 \%$ reduction in radi- ation dose can be expected without compromise in image quality dose.

Another major drawback of 4-detector-row MSCT angiography is the impaired temporal resolution resulting from its 250-ms acquisition time. In patients with heart rates of more than 80 beats/min, this leads to artifacts, especially in the distal segments of the coronary arteries. When possible, $\beta$-blockers or other negative chronotropic drugs should be administered before the $\mathrm{CT}$ investigation to reduce heart rate and limit motion artifacts, as has been shown in previous studies ${ }^{10,14}$ and in our present study. Lack of cooperation during MSCT scans, especially in younger patients, led to suboptimal image quality and reduced the diagnostic performance of the test. Thus, sedation should be mandatory in pediatric patients to limit motion artifacts.

Magnetic resonance imaging (MRI) could be an alternate modality for the diagnosis of stenosis or other anomalies in the proximal segments of the coronary arteries. ${ }^{15,16}$ However, diagnostic accuracy varies substantially according to the different studies ${ }^{17}$ and this technique is still hampered by a low spatial resolution and a long scan time compared with that of MSCT, ${ }^{18}$ thus limiting routine clinical application of this technique. New techniques ameliorating MRI are being developed, such as new coils, contrast agents, and acquisition methodologies, and must continue to improve over time for MRI to be considered as a noninvasive, non-X-ray technique that offers an alternative to MSCT for the detection of coronary complications after the ASO.

\section{Study Limitations}

We acknowledge the following limitations of the present study. First, the major limitation of this study is the relatively small number of patients. Thus, our results require further validation with more children undergoing the ASO.

Second, analyses only concern the ostial and proximal segments of the coronary arteries. However, it must be noted that delayed coronary lesions after ASO occur exclusively at the ostial segments, proximal segments, or both. ${ }^{2}$ The latest generation of MSCT scanners with 16 detector rows, 32 detector rows, and, recently, 64 detector rows, ${ }^{11}$ which allow thinner slices and faster rotation aided by advanced interactive and high-speed postprocessing application, should improve image quality and diagnostic accuracy in detecting more distally coronary segments, especially in patients whose heart rates are high during CT examination. However, imaging of distal coronary segments are less pertinent in our specific pediatric patients having undergone the ASO because lesions are only ostial, proximal, or both.

Third, no quantitative coronary angiography was performed, and stenoses were semiquantitatively assessed with both conventional coronary angiography and MSCT. Decision making in patients with high-grade coronary stenosis is 
difficult. In our practice these patients are subjected to further investigations to evaluate myocardial ischemia. ${ }^{19} \mathrm{In}$ this condition myocardial scintigraphy has been shown to be useful in selecting patients with coronary lesions who are candidates for myocardial revascularization. ${ }^{19}$

\section{Conclusion}

These results indicate that MSCT angiography, even using a relatively outdated 4-detector-row scanner, is fully accurate in detecting ostial coronary artery stenoses, proximal coronary artery stenoses, or both in the specific pediatric patients having undergone the ASO for TGA. Our results suggest that MSCT could be used as a screening technique for detecting coronary complications in the follow-up of ASO before having recourse to conventional angiography.

We thank Mrs Susan Concordet for her assistance in the preparation of the manuscript.

\section{References}

1. Tsuda E, Imakita M, Yagihara T, Ono Y, Echigo S, Takahashi O, et al. Late death after arterial switch operation for transposition of the great arteries. Am Heart J. 1992;124:1551-7.

2. Bonnet D, Bonhoeffer P, Piechaud JF, Aggoun Y, Sidi D, Planche C, et al. Long-term fate of the coronary arteries after the arterial switch operation in newborns with transposition of the great arteries. Heart. 1996;76:274-9.

3. Bonhoeffer P, Bonnet D, Piechaud JF, Stumper O, Aggoun Y, Villain $\mathrm{E}$, et al. Coronary artery obstruction after the arterial switch operation for transposition of the great arteries in newborns. J Am Coll Cardiol. 1997;29:202-6.

4. Blume ED, Altmann K, Mayer JE, Colan SD, Gauvreau K, Geva T. Evolution of risk factors influencing early mortality of the arterial switch operation. J Am Coll Cardiol. 1999;33:1702-9.

5. Brown JW, Park HJ, Turrentine MW. Arterial switch operation: factors impacting survival in the current era. Ann Thorac Surg. 2001;71: 1978-84.

6. Pasquali SK, Hasselblad V, Li JS, Kong DF, Sanders SP. Coronary artery pattern and outcome of arterial switch operation for transposition of the great arteries: a meta-analysis. Circulation. 2002;106:2575-80.
7. Legendre A, Losay J, Touchot-Kone A, Serraf A, Belli E, Piot JD, et al. Coronary events after arterial switch operation for transposition of the great arteries. Circulation. 2003;108(suppl 1):II186-90.

8. Achenbach S, Giesler T, Ropers D, Ulzheimer S, Derlien H, Schulte C, et al. Detection of coronary artery stenoses by contrast-enhanced, retrospectively electrocardiographically-gated, multislice spiral computed tomography. Circulation. 2001;103:2535-8.

9. Nieman K, Rensing BJ, van Geuns RJ, Munne A, Ligthart JM, Pattynama PM, et al. Usefulness of multislice computed tomography for detecting obstructive coronary artery disease. Am J Cardiol. 2002;89: 913-8.

10. Ropers D, Baum U, Pohle K, Anders K, Ulzheimer S, Ohnesorge B, et al. Detection of coronary artery stenoses with thin-slice multi-detector row spiral computed tomography and multiplanar reconstruction. Circulation. 2003;107:664-6.

11. Leschka S, Alkadhi H, Plass A, Desbiolles L, Grunenfelder J, Marincek B, et al. Accuracy of MSCT coronary angiography with 64-slice technology: first experience. Eur Heart J. 2005;26:1482-7.

12. Schmitt R, Froehner S, Brunn J, Wagner M, Brunner H, Cherevatyy O, et al. Congenital anomalies of the coronary arteries: imaging with contrast-enhanced, multidetector computed tomography. Eur Radiol. 2005;15:1110-21.

13. Jakobs TF, Becker RC, Ohnesorge B. Multislice helical CT of the heart with retrospective ECG gating: reduction of radiation exposure by ECG-controlled tube current modulation. Eur Radiol. 2002;12: 1081-6.

14. Giesler T, Baum U, Ropers D, Ulzheimer S, Wenkel E, Mennicke M, et al. Noninvasive visualization of coronary arteries using contrastenhanced multidetector CT: influence of heart rate on image quality and stenosis detection. AJR Am J Roentgenol. 2002;179:911-6.

15. Kim WY, Danias PG, Stuber M, Flamm SD, Plein S, Nagel E, et al. Coronary magnetic resonance angiography for the detection of coronary stenoses. N Engl J Med. 2001;345:1863-9.

16. Post JC, van Rossum AC, Bronzwaer JG, de Cock CC, Hofman MB, Valk J, et al. Magnetic resonance angiography of anomalous coronary arteries. A new gold standard for delineating the proximal course? Circulation. 1995;92:3163-71.

17. Peebles CR. Non-invasive coronary imaging: computed tomography or magnetic resonance imaging? Heart. 2003;89:591-4.

18. Budoff MJ, Achenbach S, Duerinckx A. Clinical utility of computed tomography and magnetic resonance techniques for noninvasive coronary angiography. J Am Coll Cardiol. 2003;42:1867-78.

19. Acar P, Maunoury C, Bonnet D, Sebahoun S, Bonhoeffer P, Saliba Z, et al. Comparison of myocardial perfusion single-photon emission computed tomography with coronary artery angiography after arterial switch operation. Am J Cardiol. 2001;87:1425-7. 